\title{
La representación del autismo en las narrativas de fan fiction.net. los espacios de afinidad como oportunidad para la negociación de sentido
}

Recibido: 2017-01-19

Enviado a pares: 2017-02-14
Aprobado por pares: 2017-04-04

Aceptado: 2017-04-25

DOI: 10.5294/pacla.2017.20.4.5

Para citar este artículo / to reference this article / para citar este artigo Lugo, N., Melón, M.E. \& Castillo M. C. 2017. La representación del autismo en las narrativas de fan fiction. net: los espacios de afinidad como oportunidad para la negociación de sentido. Palabra Clave 20(4), 948-978. DOI: 10.5294/pacla.2017.20.4.5

\section{Resumen}

La representación del autismo en los medios masivos ha retenido a lo largo del tiempo estereotipos que parafrasean el discurso médico. Este artículo se plantea como objetivo indagar si existen representaciones alternativas en espacios de afinidad como fan fiction.net. Para explorarlo, se realizó un estudio mixto compuesto de un análisis cuantitativo sobre 197 fanfictions encontrados en la plataforma en noviembre de 2016, con la etiqueta autism, en los cuales se midieron indicadores de popularidad de lectura, como suscriptores, favoritos y número de comentarios. Posteriormente, se realizó un análisis cualitativo del texto más relevante de acuerdo con la popularidad y el contenido. Dicho análisis se enfocó en el fan fiction Different, Not Defective perteneciente al fandom de Harry Potter y el análisis de los 863 co-

orcid.org/0000-0002-1334-7914. Tecnológico de Monterrey, México.nlugo@itesm.mx

2 orcid.org//0000-0002-9355-906X. Tecnológico de Monterrey, México. mmelon@itesm.mx

3 orcid.org/0000-0002-2892-9079. Tecnológico de Monterrey, México. ccastill@itesm.mx 
mentarios al texto. Los resultados muestran que las plataformas digitales pueden funcionar como espacios inclusivos donde terapeutas, familiares y personas dentro del espectro autista incorporan formas alternativas de representación. Las conclusiones indican que estos espacios tienen un gran potencial para negociar el sentido del autismo, lo cual podría extenderse a otras discapacidades. Una de las aportaciones del presente estudio es proveer una metodología que permita estudiar otros fandom u otras discapacidades. Se sugiere seguir explorando cómo el fan fiction en internet puede coadyuvar en la creación de sentidos emergentes acerca de grupos inadecuadamente representados.

\section{Palabras clave}

Fan fiction; autismo; representación mediática; Harry Potter; discapacidad; discurso médico; negociación (Fuente: Tesauro de la Unesco). 


\section{Representation of Autism in fanfiction. net Narratives: Affinity Spaces as an Opportunity for Negotiating Meaning}

\section{Abstract}

Representation of autism in mass media has retained stereotypes that paraphrase medical discourse over time. This paper aims to investigate about the existence of alternate representations in affinity spaces like fanfiction. net. For this purpose, we conducted a mixed composite study of a quantitative analysis of 197 fanfictions found in the platform in November 2016 under the tag autism, in which reading popularity indicators such as subscribers, bookmarks and number of comments were measured. We then conducted a qualitative analysis of the most relevant text according to popularity and content. This analysis focused on the fanfiction Different, Not Defective, from the Harry Potter fandom, and the analysis of the 863 comments. Results show that digital platforms can function as inclusive spaces where therapists, family members and individuals within the autistic spectrum incorporate alternative forms of representation. The conclusions indicate that these spaces have a great potential to negotiate the meaning of autism, which could extend to other disabilities. One of the contributions of this study is that it provides a methodology for studying other fandom or other disabilities. We suggest continuing to explore how fan fiction on the internet can contribute to the creation of emerging meanings about groups that are inadequately represented.

\section{Keywords}

Fan fiction; autism; media representation; Harry Potter; disability; medical discourse; negotiation (Source: Unesco Thesaurus). 


\section{A representação do autismo nas narrativas de fan fiction.net: os espaços de afinidade como oportunidade para a negociação de sentido}

\section{Resumo}

A representação do autismo na mídia em massa tem retido ao longo do tempo estereótipos que parafraseiam o discurso médico. Este artigo é abordado com objetivo indagar se existem representações alternativas em espaços de afinidade como fan fiction.net. Para explorá-lo, se realizou um estudo misto composto de uma análise quantitativa sobre 197 fanfictions encontrados na plataforma em novembro de 2016, com a etiqueta autism, nos quais se mediram indicadores de popularidade de leitura, como assinantes, favoritos e número de comentários. Posteriormente, realizou-se uma análise qualitativa do texto mais relevante de acordo com a popularidade e o conteúdo. Esta análise teve como foco o fan fiction Different, Not Defective pertencente ao fandom de Harry Potter e à análise dos 863 comentários no texto. Os resultados mostram que as plataformas digitais podem funcionar como espaços inclusivos onde terapeutas, familiares e pessoas dentro do espectro autista incorporam formas alternativas de representação. As conclusões indicam que estes espaços têm um grande potencial para negociar o sentido do autismo, o qual poderia ampliar-se a outras incapacidades. Uma das contribuições do presente estudo é proporcionar uma metodologia que permita estudar outrosfandom ou outras incapacidades. Sugere-se continuar explorando como o fan fiction na internet pode coadjuvar na criação de sentidos emergentes sobre grupos inadequadamente representados.

\section{Palavras-chave}

Fan fiction; autismo; representação mediática; Harry Potter; incapacidade; discurso médico; negociação (Fonte: Tesauro da Unesco). 


\section{Introducción}

En este artículo, se analiza la representación del autismo, condición médica que se ha redefinido históricamente de según el avance en investigación y diferentes corrientes y visiones de la psicología y la medicina. El discurso científico llegó al límite de indicar que el autismo era resultado de la falta de amor de las madres hacia sus hijos en la infancia temprana, lo cual provocaba una desconexión con el mundo. Esta fue una de las primeras hipótesis de Leo Kanner, quien descubrió y nombró esta condición neurológica en 1943. Dicha hipótesis ha sido rechazada por la comunidad científica contemporánea (Ramachandran y Oberman, 2006; Osteen, 2010). Sin embargo, desde que se le nombró y definió a la fecha, las personas con autismo han sido objeto de muchas representaciones inadecuadas que afectan su inclusión social y autoestima.

De acuerdo con Hall (2010), la representación actúa como una imposición discursiva que es imperceptible para los sujetos por dos razones: la primera, por el carácter estructural y colectivo de la ideología que deriva en la segunda razón, su naturalización. Dicho de otro modo, no somos conscientes de nuestra propia ideología, porque nos incrustamos en la vida cotidiana dentro de las estructuras que han conformado el grupo social al que pertenecemos. Por ello, es importante cuestionar las representaciones hegemónicas. Si bien es común pensar que las representaciones basadas en medios son las que más influyen en alimentar prejuicios, el campo de disability studies da cuenta de cómo los prejuicios que se originan en el campo de la medicina fomentan la validación de prejuicios en los medios.

El propósito específico de este artículo fue indagar sobre si el autismo se representa en el fan fiction, y de ser así, por quién y cómo, así como qué sucede con la retroalimentación de los lectores a lo largo de la escritura de los textos. El artículo presenta un cruce entre dos perspectivas: se nutre de los disability studies y se incorpora a la tercera ola de los fan studies, que busca contribuir a los estudios culturales de forma global (Thomas, 2011).

La mayoría de los estudios de la representación del autismo se ha concentrado en series televisivas o películas. El fandom es un espacio de 
afinidad escasamente contemplado en los artículos de representación con el tema de la discapacidad (Black, 2008). El caso de fan fiction.net, la plataforma con más textos de fan fiction en internet, es un caso digno de estudio, ya que, mientras coincide con la representación ficcional de personas con trastorno del espectro autista (TEA) representadas en películas o series televisivas, los fanfictions suelen diferir en que presentan una relación más equitativa y un cambio de roles entre enunciadores y destinatarios: los autores pueden ser a su vez retroalimentadores y la obra se retroalimenta mientras está en construcción.

La perspectiva en este artículo coincide con la que Osteen (2010) llama empathetic scholarship para referirse a la combinación de una investigación rigurosa y el estudio del tema con una actitud de diálogo y validación hacia la experiencia de las personas con autismo o sus familiares.

En el marco teórico, se explica quiénes han determinado cómo se representa el autismo y posteriormente se explora cómo este se ha representado en los medios masivos de comunicación. Después se presenta el proceso sociocultural de escritura y lectura en el fan fiction, lo cual es esencial para comprender cómo esto influye en la construcción de sentido.

En el método se describe cómo se llevó a cabo el estudio mixto y en qué consistieron cada una de las fases del análisis cuantitativo y cualitativo. Finalmente, en los hallazgos, se incluye lo más relevante del análisis cuantitativo y cualitativo para llegar a la conclusión en la que se confirma la hipótesis de que en los espacios de afinidad como fan fiction.net se pueden encontrar representaciones alternativas del autismo.

\section{Marco teórico}

\section{El concepto de autismo: de sus representaciones en la ciencia al concepto de neurodiversidad}

Hasta hace muy poco la descripción del autismo se ha basado en la tríada de déficits propuesta por Wing, a su vez basada en el trabajo de Kanner y Asperger. Esta apunta a una visión de déficit y anormalidad. Se compone 
de déficit en la interacción social, déficit en la comunicación y déficit en la imaginación (Brownlow, 2010).

Para diagnosticar a una persona dentro del espectro, en varios países se recurre al Diagnostic and Statistical Manual of Mental Disorders (DSM) a pesar de no ser una enfermedad psiquiátrica o mental, sino una condición neurológica. Esto se debe en parte a la falta de otras formas de diagnosticarlo, además de un análisis basado en observación de la conducta. Hasta el DSM-4 se dividía en autismo "de alto funcionamiento" o "bajo funcionamiento", Asperger y trastorno generalizado del desarrollo. De acuerdo con Schwarz (2010), esta división ha causado una falsa dicotomía, pues muchas personas con autismo pueden tener un grado de discapacidad en algunos aspectos, pero no en otros. Además, puede crear profecías autocumplidas: ser etiquetado de "bajo funcionamiento" crea bajas expectativas que promueven desesperanza o pasividad. Las etiquetas, el lenguaje y los criterios de diagnóstico son importantes, porque tienen influencia en el comportamiento y la percepción de la gente que pretenden evaluar (Quirici, 2015).

En el DSM-5, la versión más reciente, el trastorno del espectro autista solo se clasifica como tal, una variación, probablemente en un intento de reconocer esta diferencia. Sin embargo, hay desacuerdo entre organizaciones, padres, etc., sobre si esto fue acertado o no, especialmente cuando puede citarse para alegar que no se requieren apoyos económicos para el tratamiento.

El discurso médico contemporáneo no es uniforme. Entre los investigadores líderes en la investigación en autismo existen posturas diversas. Por ejemplo, un reconocido neurólogo, V. S. Ramachandran, y su grupo de investigación han hecho importantes avances en descubrir el rol de las neuronas espejo en los síntomas del autismo. Sin embargo, en un artículo de 2006, usan palabras como "enfermedad", constantemente apuntan a las "disfunciones" y el "déficit" de las personas con autismo y afirman haber descartado de los sujetos de investigación a "niños de autismo de bajo funcionamiento", debido a que su "discapacidad intelectual" podría desviar los resultados. En contraste, Dawson, Soulières, Gernsbacher y Mottron 
(2007) probaron que el autismo no siempre implica discapacidad intelectual y demostraron que el tipo de prueba modificaba significativamente los resultados. Concluyeron que la inteligencia de las personas con autismo se ha desestimado. El grupo de investigación y el laboratorio en neurociencia cognitiva dirigido por Laurent Mottron se enfoca en estudiar y cuestionar muchos de los mitos respecto del autismo.

Osteen (2010) señala que personas con autismo y diagnosticados con Asperger, quienes se autoproclaman "aspies", han tomado las riendas de la representación declarando que no están enfermos y no necesitan ser curados. La definición de neurodiversidad fue acuñada por Judy Singer y busca presentar el autismo más como una diferencia que como un déficit. Brownlow (2010) explica que esto es coherente con los estudios de discapacidad desde un modelo social; estudios que apuntan a que la discapacidad es biológica, pero el verdadero problema es la falta de integración de dicha discapacidad en la sociedad. Aunque originalmente la idea de la neurodiversidad fue un concepto concebido por gente con autismo, ha sido acogida por otros grupos en su reconcepción de déficit versus diferencia. Esta idea no propone puestos binarios, sino un espectro amplio de constituciones neurológicas en los que el autismo es uno (Brownlow, 2010, p. 253). Esta tendencia, sin embargo, posiblemente ha profundizado una división en la cultura del autismo, la brecha entre "alto" y "bajo funcionamiento" y dejado sin voz a las personas catalogadas en el segundo grupo (Osteen, 2010).

\section{Representación del autismo: de los medios masivos a internet}

De acuerdo con Sarrett (2011), mientras las investigaciones contemporáneas permiten una comprensión más profunda del autismo, los supuestos sobre este se han mantenido, ya que se observa desde sus representaciones y el público hereda una ideología sobre lo que significa. El autor analiza las imágenes con las que se representa a los niños con autismo en medios impresos comparando la década de 1960 con la primera década del siglo XXI. Descubre que las metáforas raíz y tropos comunes sobre el autismo se han mantenido, sobre todo la de la persona fragmentada, no completa. Jones y Hardwood (2009) analizan la representación del autismo en la prensa aus- 
traliana entre 1996 y 2005 . Concluyen que se mantiene una cantidad muy limitada de información basada en hechos y los estereotipos que predominan es que las personas con autismo son incontrolables, peligrosas, no amadas o tratadas muy mal. Por su parte, Belcher y Maich (2014) analizaron cómo se representa el autismo en diversos medios, como libros para niños, novelas populares, televisión comercial y películas comerciales entre 2006 y 2012. Escogieron para ello una muestra de veinte productos. Encontraron que las novelas tienden a presentar el TEA de una manera compleja con un contexto familiar y comunitario más auténtico; los libros ilustrados tratan de explicar el autismo desde una perspectiva clínica y no siempre adecuada para fomentar la inclusión educativa. En la televisión, se tiende a representar a las personas con autismo como genios y héroes que conquistan retos aparentemente imposibles.

Otra de las representaciones comunes en la televisión o el cine es el savant. Si bien este síndrome está estrechamente vinculado al autismo, solo $10 \%$ de las personas con TEA lo presenta. Originalmente, este síndrome era llamado de los "genios-idiotas". Esto se ha corregido y se aplica para personas con un talento sobresaliente, normalmente en áreas como la memorización visual o de datos, el dibujo o la ejecución musical (Dawson et al., 2007). Según Quirici (2015), la expectativa de genialidad se debe a representaciones populares como Rainman y The Big Bang Theory. La investigadora apunta a cómo se asume que la genialidad de los savants es resultado de un cerebro anormal y, por tanto, las personas con autismo no reciben crédito por sus talentos. En este caso, según su "genialidad", las personas con autismo son vistas como "el otro", aislados y deshumanizados tras el alegato de genialidad, lo cual permite admirar a los savants como espectáculo y no envidiarlos, porque su talento implica un costo muy alto.

Mientras todos estos estudios basados en diferentes medios coinciden en general en que los medios promueven una imagen estereotipada del autismo, Acosta-Alzuru (2013) en su estudio de Micaela, un personaje con Asperger que aparece en una telenovela venezolana, describe el proceso de investigación del creador de la telenovela y la aprobación de la caracterización expresada por la comunidad venezolana con Asperger a través de foros en internet. 
Internet parece haber ampliado la posibilidad de divulgación y la diversidad de voces gracias a las asociaciones civiles o personas con autismo y activistas como Temple Grandin, editoriales que venden sus obras en internet como Jessica Kingley o al potencial de la cultura participativa (Jenkins, 2006), ya que, gracias a las experiencias de las personas con TEA o sus familias, se cuentan historias desde un punto de vista experiencial. Pero no se puede generalizar: al comparar medios masivos con sitios de organizaciones en internet de asociaciones de padres o asociaciones civiles, Stevenson, Harp y Gernsbacher (2011) descubrieron que, aun sin una mala intención, en ambos casos hay una infantilización del autismo, puesto que los adultos con TEA no están representados y, debido a la falta de representación, puede disminuir el apoyo necesario para su inclusión social.

\section{Los procesos de escritura, lectura y retroalimentación en el fan fiction}

Los fanfictions publicados en internet son un tipo de escritura amateur que se basa en argumentos, personajes o mundos narrativos preexistentes que provienen de los textos mediáticos favoritos de los fans (Black, 2008; Parrish, 2007). Los fans se motivan a escribir y compartir estos textos debido al vínculo afectivo que tienen con el mundo narrativo y sus personajes (Guerrero-Pico, 2016; Samutina, 2016). En el campo de los fan studies, dicho mundo es llamado canon (Karpovich, 2006). Las ficciones que se generan en torno a él con sus propias convenciones enriquecidas por la comunidad de fans son el fanon.

En el proceso de escritura, hay una mezcla de estrategias para modificar la ficción: 1) las estrategias narrativas como extender o comprimir el mundo narrativo en el nivel espacial, temporal o de los personajes (Guerrero-Pico, 2016) y 2) los recursos y las estrategias culturales que usan los fans para hacer que esas ficciones tengan sentido desde su propia experiencia, por ejemplo la refocalización, el reordenamiento moral, la intensificación emocional y la erotización (Jenkins, 1992). La escritura de un texto de fan fiction implica una manera subjetiva de apropiarse personal y culturalmente de un mundo narrativo, para lo cual se utilizan diferentes recursos personales y estrategias narrativas (Lugo, 2016). 
Una de las características de este tipo de escritura cuando es publicada en internet es que es procesual (Hellekson y Busse, 2006). Se publica capítulo a capítulo, por lo cual los lectores pueden retroalimentar durante la elaboración de la obra.

Según Thomas (2011), atender los procesos de análisis textual con un enfoque en el proceso de producir y diseminar historias puede aclarar cómo los fans interactúan e interpretan los mundos narrativos, puesto que basarse solo en el estudio del texto deja muchas preguntas sin responder. La autora agrega que la investigación debe enfocarse en la pregunta de qué hacen los lectores y las audiencias con esos mundos: "cómo los habitan, transforman y los hacen suyos" (p. 7). Además de poder retroalimentar en cuanto un capítulo es publicado, en las plataformas en las cuales se publican fan fiction, todos los usuarios pueden cambiar de rol: ser escritores y lectores (Jenkins, 1992; Fiske, 1992; Karpovich, 2006). Hay un mayor equilibrio entre el autor y el receptor. Si los escritores del fanfic pueden considerarse poachers (Jenkins, 1992), dado que los fans son activos y, más allá de la interpretación, reescriben, resignifican y se apropian así del mundo narrativo del canon, puede decirse que, a su vez, los lectores del fan fiction tienen el potencial de desempeñar el mismo papel y ser, a su vez, poachers de cada fanfic, ya que con su propia retroalimentación tienen influencia en la escritura de los siguientes capítulos. Igualmente, son poachers si deciden retomar textos de escritores de fan fiction, y escribir a partir de ellos. Esto es especialmente significativo en el ámbito de la representación. Debido a que el proceso de significación en estas narrativas se discute y negocia durante su elaboración, estas historias difieren de los reportajes periodísticos, las películas y las obras literarias que entran en el proceso de circulación cultural, una vez que han sido concluidas.

\section{Método}

Castillo y Meneses (2015), basadas en Moretti (2015), proponen una visión de mirada distante a cercana para analizar los fenómenos de la comunicación en medios digitales. Esta mirada fundamenta una aproximación metodológica en la que se observen y validen las grandes cantidades de datos que se acumulan en internet, a la vez que se reconozcan dichos datos como 
construcciones culturales que pueden interpretarse, en un primer momento, de forma distante para luego realizar análisis cercanos de corte cualitativo. Esta investigación toma esa postura. El diseño de investigación fue en cierta medida emergente: el análisis cuantitativo constó de las tres primeras fases y el análisis cualitativo de dos más, es decir, las fases cuatro y cinco. Los resultados de cada fase orientaron la siguiente. A continuación, se describen las fases. El análisis cuantitativo cubrió de la primera a la tercera fase y el análisis cualitativo la cuarta y quinta fases.

\section{Mirada distante: análisis cuantitativo}

- Primera fase: exploración de la difusión y la recepción a través de datos cuantitativos: follow, review y suscribers.

- Segunda fase: visualización de datos de popularidad y de metadatos de cada una de las 197 historias.

- Tercera fase: selección de corpus cualitativo según los datos revelados en la visualización. Different, Not Defective: uno de los dos fanfictions más populares y con más comentarios.

\section{Mirada cercana: análisis cuantitativo}

El análisis cualitativo, incluyó las últimas dos fases de la investigación:

- Cuarta fase: análisis narrativo y de la representación de Different, Not Defective.

- Quinta fase: análisis cualitativo de 863 comentarios a la historia Different, Not Defective.

\section{Mirada distante: análisis cuantitativo}

\section{Primera fase: exploración de la difusión y la recepción a través de datos cuantitativos}

La primera fase fue descubrir cuánto del contenido de los fanfictions de la plataforma seleccionada se refiere a autismo. Se seleccionó esta plataforma, porque es la que recoge más fanfictions en el ámbito internacional. Para definir el corpus de análisis, se buscó la etiqueta "autism", ya que la mayor parte de las publicaciones son en inglés. Esta búsqueda dio como resultado 197 
textos. Se creó una tabla con los metadatos que ofrece la plataforma para cada historia: número de palabras, capítulos, fecha, género, etc. En la tabla, se agregaron otros datos que no están disponibles, sino que requieren una lectura de la introducción de los textos. Estos fueron:

1. El canon: Harry Potter, Twilight, Pokemon, etc.

2. Relación del escritor con el tema. En este caso, se indagó sobre si la introducción de la historia contaba con alguna declaración explícita del autor sobre su interés en el tema del autismo o su relación con este: persona con TEA, familiar, interesado, etc. Los datos cuantitativos más relevantes fueron los indicadores de recepción que apuntaban a la reacción de los lectores desde tres perspectivas:

a. Review: los comentarios que cualquier retroalimentador puede proporcionar al autor.

b. Favorite: los usuarios tienen la posibilidad de marcar como favorito al autor o al fanfic en específico. En este caso, se contabilizaron los favoritos de la ficción.

c. Follow: esta es una suscripción a la historia para ser notificado cuando existan más capítulos disponibles de la obra.

Figura 1. Género

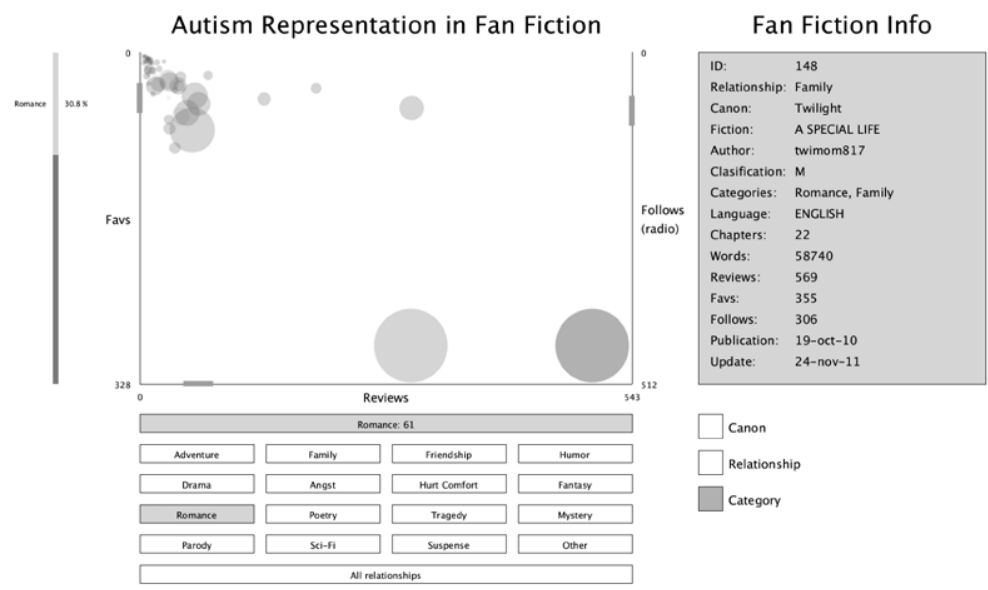

Fuente: Elaboración propia. 
Para encontrar los textos más leídos desde la recepción, se contabilizaron las participaciones de los retroalimentadores. A cada review, favorite o follow se le asignó un valor igual (1), puesto que, aunque el comentario es cualitativo y requiere mayor esfuerzo del retroalimentador, indica que una historia está entre las favoritas o suscribirse para seguirla son una muestra igualmente valiosa. Esto intenta validar la participación de los diferentes tipos de usuarios. Guerrero-Pico (2016) explica que la participación de un comentador no tiene por qué ser más valiosa que la de un lector sino diferente. El lurker, como se considera en el fandom al lector que explora sin comentar, es un participante silente, pero que deja marca en su interacción en el texto. Indicar que un texto es favorito o suscribirse sin comentar es una manera de ser lurker que permite ser cuantificada. Por otro lado, si bien puede haber retroalimentadores que indiquen una misma historia como favorita y además la comenten, se contabilizaron como dos participaciones sin importar si son del mismo usuario.

\section{Segunda fase: visualización de datos}

Según los datos obtenidos de la plataforma, en la lectura de las introducciones de los textos y con la asignación de puntos a cada participación, se programó en el lenguaje Processing una visualización de datos que permitió identificar relaciones y características de las ficciones. Se decidió visualizar la información que las investigadoras consideraron más relevante: 1) indicadores de recepción: review favorite y follow, 2) relación de los autores con el tema y 3) género de las ficciones indicado por el autor.

En la visualización, cada ficción está representada por un círculo y su tamaño está determinado por el número de seguidores. El número de comentarios y de favoritos ubican espacialmente las diferentes ficciones. También se ha visualizado información sobre el número de ficciones y el porcentaje que representan. En los hallazgos se revelará la información de la visualización. Esta se puede consultar en http://piedramuda.com/fanfictionvis/

\section{Tercera fase: selección de corpus cualitativo}

La visualización destacó las ficciones con mayor número de participaciones en los tres indicadores de recepción: 1) review, 2) favorite y 3) follow. 
Entre ellos, se distinguen tres ficciones que cuentan con mayor número de participaciones de los retroalimentadores. La recepción esta polarizada. Pocos textos se leen mucho.

Para el análisis cualitativo, se escogió un solo texto a fin de analizar a fondo cómo se representaba el autismo tanto en la ficción como en las reacciones de los retroalimentadores. El texto seleccionado se escogió entre los dos textos con mayor índice de recepción de acuerdo con los datos arrojados por el análisis cuantitativo. Según una primera lectura de la ficción y los comentarios, se descubrió qué texto era más propicio para el análisis: Different, Not Defective del canon Harry Potter.

\section{Mirada cercana: análisis cualitativo}

Este análisis cubrió la cuarta y quinta fase. Se dividió en 1) un análisis narrativo y 2) un análisis de la representación en los comentarios de los retroalimentadores.

\section{Cuarta fase: análisis narrativo}

Como categorías analíticas previas, solo se tenían "narrativa", "representación” y "autismo", pues se intentaba descubrir cómo se representa el autismo en una ficción derivada. Se hizo una codificación de primer y segundo nivel (Hernández, Fernández y Baptista, 2014). De esta resultaron las estrategias a través de la cuales el tema se representaba: la caracterización, el tipo de narrador y el espacio-mundo narrativo. Por tanto, se descubrió que el texto se podía analizar bajo los conceptos básicos del análisis literario, ya que la representación se tejía a través de todos estos elementos.

\section{Quinta fase: análisis de los comentarios de los retroalimentadores}

Estos se dividieron por cada uno de los quince capítulos del fan fiction. Se hizo una primera lectura para descubrir los temas generales en la retroalimentación, los cuales se dividían en reacciones emocionales ante la historia, narrativos o sobre el autismo. Posteriormente, se recurrió a codificar los comentarios relacionados con "autismo", los cuales compartían múltiples códigos como "tipo de relación con el tema”, "aprobación”, "identificación del lector con el autor", etc. 


\section{Hallazgos y discusión}

\section{Hallazgos del análisis cuantitativo}

\section{Quiénes escriben}

Se descubrió que los autores tienen diferentes roles en relación con el espectro autista: sí mismo (10.1\%), es decir, declaran estar dentro del espectro, afirman ser familiares $(7.1 \%)$, voluntarios $(3.5 \%)$, amigos $(4.5 \%)$ interesados $(5.6 \%)$, maestros $(2.5 \%)$, profesionales (1\%). En su gran mayoría, no especifican ni declaran ninguna postura ante el tema o ninguna relación con una persona con autismo $(65.2 \%)$. Sin embargo, en la lectura rápida de algunos de los textos, se puede distinguir que hay personas que, si bien no declaran una relación con el TEA, están familiarizadas con personas que lo tienen. Esto se nota, dada la descripción tan detallada o la caracterización o datos que incluyen en el fic.

Figura 2.

Relación con el autismo. Sí mismo

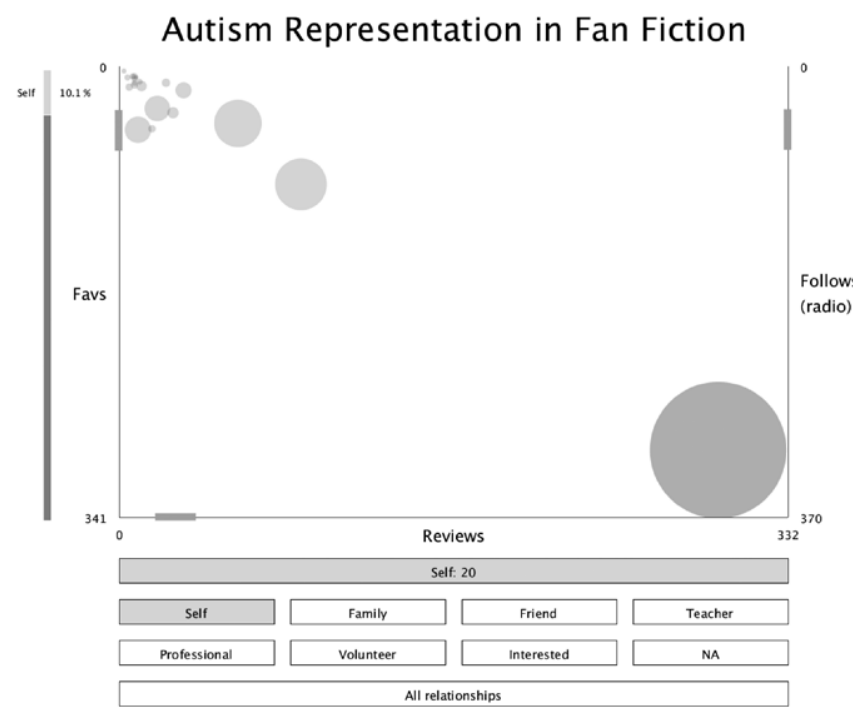

Fan Fiction Info

\begin{tabular}{ll|}
\hline ID: & 147 \\
Relationship: & Self \\
Canon: & Twilight \\
Fiction: & COPING WITH CHANGE \\
Author: & The Plasma \\
Clasification: & T \\
Categories: & Romance \\
Language: & ENGLISH \\
Chapters: & 27 \\
Words: & 61812 \\
Reviews: & 339 \\
Favs: & 355 \\
Follows: & 306 \\
Publication: & 10 -dic-09 \\
Update: & 13 -jun-14 \\
\hline
\end{tabular}

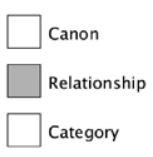

Fuente: Elaboración propia. 


\section{Canon}

La mayor cantidad de textos con relación al TEA pertenecen al canon de Twilight (16.2 \%) y de Harry Potter (7.1 \%). Exceptuando estos, la mayoría se reparte en cánones muy diversos, lo cual coincide con que los cánones Twilight y Harry Potter (HP) son los más leídos, y sobre los que más se han indicado review, favorite y follow. Otro descubrimiento relevante es que existe $3 \%$ de ficciones originales no relacionadas con ningún canon, sino solo con la etiqueta autism.

\section{Géneros}

Los autores categorizan sus historias en varios. El género más común es "romance" con $30.5 \%$, seguido de "familia" con $28.3 \%$, "drama" y "Hurt/ Comfort” comparten $22.2 \%$ y amistad $19.2 \%$. Es decir, los textos relacionados con autismo exploran relaciones interpersonales.

\section{Hallazgos del análisis cualitativo}

\section{Análisis narrativo de Different, Not Defective}

Premisa, argumento y género

El fan fiction Different, Not Defective es una historia que cuenta con quince capítulos. Comenzó a escribirse en 2009 y concluyó en 2011 y gira alrededor de la entrada de Harry al colegio de magia Hogwarts. Los eventos presentados concuerdan con los narrados en el primer libro La piedra filosofal en el que Harry vive con su familia, quienes son familiares abusivos con él. Igual que en la ficción original, Harry es llevado al cumplir 11 años a Hogwarts. Sin embargo, en el texto, se aprecian algunas alusiones a momentos posteriores en el mundo narrativo de J. K. Rowling. El autor o autora del fanfic presenta su premisa así: “Todos decían que Harry era diferente, pero qué pasaría si sus diferencias van más allá de sobrevivir la maldición? ¿Qué pasaría si Harry tuviera autismo? ¿Cómo podría el mundo mágico reaccionar y adaptarse al niño que sobrevivió?” (Awesomelyglorius, 2009-2011).

Pertenece a la variación alternate universe (Hellekson y Busse, 2006), es decir, un texto que presenta un cambio en parte de la premisa de la historia. Dentro de los géneros de fan fiction, su autor lo cataloga como Hurt/ 
Comfort/amistad. El primero es un género específico del fan fiction que se caracteriza por que un personaje es herido, física o emocionalmente, y otro personaje lo consuela (fanfiction.net; tvtropes.org). En este caso, la amistad, más que un género, es un tema. En la historia, el personaje que sufre es $\mathrm{Ha}$ rry y el que lo consuela es el profesor Snape. Quien, en este caso, es out of character, término utilizado en el fandom para indicar que su personalidad es poco coherente con la del personaje en el canon.

En esta historia, Harry sufre porque debido a tener TEA es sujeto de bullying en la escuela. La ficción comienza en la escena en la cual los alumnos de primer año son escogidos, por el sombrero mágico, para saber a qué casa debe pertenecer. Es a través de su reacción a este evento que se introduce al lector en la condición que Harry tiene.

Caracterización de los personajes

Harry Potter es caracterizado como autístico. Su condición se manifiesta cuando reacciona ante ciertos eventos o situaciones o simplemente en su interacción con las otras personas. Así, los lectores pueden descubrir diferentes aspectos del trastorno, por ejemplo el síndrome de desintegración sensorial. En el texto, se puede observar lo mucho que afectan a Harry las luces, el brillo, los sonidos de cierto tipo y la hipersensibilidad en su piel:

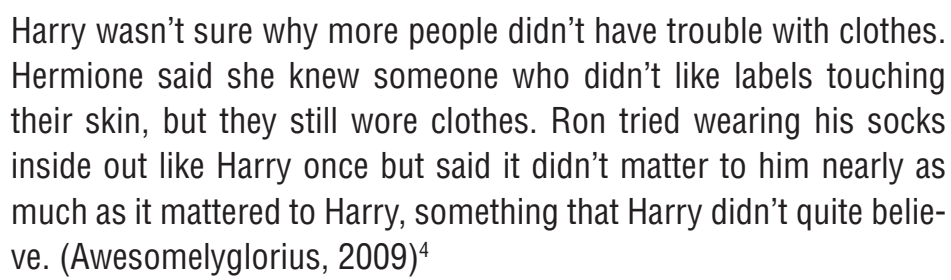

En la historia, también se evidencia cómo disfruta del sentido del tacto tocando texturas, las paredes de Hogwarts, etc. Además, se descubren otros aspectos, como la diferencia en el desarrollo motor. Como en el canon, Harry tiene 11 años. Pero en esta historia requiere ayuda para atarse

4 Harry no estaba seguro de por qué la gente no tenía problemas con su ropa. Hermione decía que conocía a alguien a quien no le gustaba cómo se sentían las etiquetas en su piel, pero aún así usaban ropa. Una vez, Ron trató de usar sus calcetines al revés como Harry, pero dijo que prácticamente no notó la diferencia, algo que Harry no podía creer. 
los cordones de los zapatos o abrocharse los botones por problemas de motricidad fina. En muchas escenas, se expresa de manera divertida cómo Harry interpreta la información de manera literal; también se nota cómo siente ansiedad ante la incertidumbre y los cambios y cómo tiene reacciones emocionales muy intensas.

Harry se caracteriza como honesto e inocente. Su descripción resulta dulce y conmovedora. Produce empatía en el lector tanto por su personalidad y caracterización como por la situación que vive. Su condición lo dota de complejidad, pero, además, el personaje va compartiendo su propio descubrimiento de lo que significa para él como individuo y para otros tener esta condición médica y cómo esto lo sitúa en el mundo. Especialmente, en el contexto de ser "El que logró sobrevivir" y de quien se tienen expectativas de ser un héroe perfecto.

Por su parte, Snape es llamado a cambiarse a sí mismo a través de la presencia de Harry en su vida, pero también es llamado a cuestionar la escuela, los profesores y transformar el entorno escolar. En las siguiente escenas, se puede apreciar este proceso personal e institucional:

Who else will protect the boy from the ignorance of our peers, Minerva? Who else will help him reach his full potential when most people think he is impossible to teach and who can't bother themselves to try?

I want to help him tie his shoes and tie his tie and learn sign language and encourage his love of chemistry and potions. I want to be there when he gets his magic to work how he wants it to for the first time, and all the times after that. (Awesomelyglorius, 2009) ${ }^{5}$

5 ¿Quién más protegerá al niño de la ignorancia de nuestros colegas, Minerva? ¿Quién más lo ayudará a alcanzar todo su potencial cuando la mayoría de los profesores considera que es imposible que aprenda y quién más se molestará en intentar enseñarle?

Quiero ayudarlo a atarse los zapatos, a hacerse el nudo de la corbata, quiero aprender lenguaje de señas. Quiero fomentar su pasión por la química y las pociones. Quiero estar ahí cuando logre que su magia funcione como lo quiere por primera vez y de ahí en adelante. 
Viaje del héroe y arquetipos

Si la historia se analiza según el paradigma del viaje del héroe (Campbell, 1959; Vogler, 2007), se puede observar que hay dos viajes. Uno es el que vive Harry. Este es más interno, es decir, la lucha de Harry es aceptar y validarse a sí mismo y fortalecerse. Hasta donde llega la historia, Harry se ve liberado de su familia abusiva y no tendrá que regresar a casa de los Dursley, pero apenas está descubriéndose a sí mismo y todavía no se observa del todo empoderado, sino en proceso de hacerlo; apenas inicia en su viaje de transformación. El viaje de Snape es interno y externo. Es llamado a cambiarse a sí mismo y a cambiar Hogwarts en cuanto a su aceptación de Harry, e incluso en cuanto a la sensibilización sobre el TEA, las políticas de inclusión educativa y el rol de los profesores en ello.

Narrador y punto de vista

Harry es presentado a través de tres puntos de vista: 1) desde el suyo, que se manifiesta en monólogos internos y revelan las sensaciones y emociones del personaje y las reflexiones que hace sobre su identidad y autoconcepto; 2) un narrador omnisciente; y 3 ) la mirada del profesor Snape. Esto es un recurso valioso en la representación, porque permite al lector distinguir el mundo interno del personaje y cómo se le ve desde el exterior. Pero en todos los casos la mirada es de aceptación. El punto de vista narrativo conduce el punto de vista sobre aceptar a una persona con TEA. En una de las escenas, se puede observar cómo el profesor Snape aprende a comprender, validar y respetar la condición neurológica de Harry. Snape parece un mentor no solo para Harry sino para el lector:

Snape: "I want to apologize to you, Mr. Potter, for silencing your hands."

Harry gaped. Then stared at his hands. Hands didn't talk.

Harry: “I don't understand. My hands didn't say anything.

Snape: "Yes, well, I apologize for having stopped you from expressing yourself with your hands in the past, and I shall endeavor to refrain from doing so in the future."

Harry: Right. The flapping and twitching and shaking and flicking, that's what he meant by 'talking,' not actual words. Stimming. "Stimming. Yes, that's the Muggle pathology for what is usually a harmless, albeit different, physical expression of emotion. And focus. 
Helps me focus and calm down. Otherwise I feel anxious and out of myself, out of myself like I don't know where I am in space and time. Out of myself. (Awesomelyglorius, 2009) ${ }^{6}$

Hogwarts como espacio y mundo narrativo

Hogwarts, como contexto narrativo, conserva las propiedades del canon. Los alumnos son clasificados en casas. Harry pertenece a Griffyndor y sus mejores amigos son Hermione y Ron. Los personajes principales, excepto Snape, tienen los mismos roles. La profesora McGoniggal imparte transformaciones y Dumbledore es el rector. Los alumnos tienen clases de pócimas y practican el deporte Quidditch.

En la historia, aparece la sala de los menesteres, habitación que en el canon se convierte en lo que el personaje necesita. En este caso, se convierte en un cuarto sensorial lleno de colores azules, texturas, y una hamaca. Harry recurre a este cuarto después de haber sido engañado por los alumnos que no solo se burlan de él sino que llegan al abuso físico.

Hogwarts representa la escuela como institución en conflicto, por lo que significa la inclusión educativa. Se evidencian las distintas actitudes hacia la diferencia de Harry. La ficción pone en tensión valores narrativos, es decir, los valores opuestos que generan tensión en una narrativa y valores humanos. El conflicto de fondo para Harry y para la escuela es la no aceptación versus la aceptación.

Estrategias narrativas y tratamiento del tema

El fan fiction como tal puede cumplir con las expectativas de los lectores en un espacio de afinidad como fanfiction.net, en el que se esperan ficciones que jueguen con el mundo original dentro de los estándares

6 Snape: "Quiero disculparme con usted señor Potter, por silenciar sus manos”.

Harry quedó boquiabierto y volteó a mirarse las manos. "No lo entiendo. Mis manos no dijeron nada”.

Snape: "Sí, bueno. Me disculpo por haberlo detenido al expresarse con sus manos en el pasado. Me comprometo a no hacerlo en el futuro".

Harry: Ya. El aleteo y los temblores y los movimientos rápidos. A eso se refería con "hablar", no palabras literalmente. "Comportamientos y movimientos repetitivos" es la patología muggle para lo que normalmente es una expresión física de emoción no dañina, aunque diferente. Y enfoque. Me ayuda a enfocarme y a calmarme. De otra manera, me sentiría ansioso, fuera de mí, como si no supiera dónde estoy en el tiempo y el espacio. Fuera de mí. 
culturales establecidos por el fandom: 1) es coherente con el canon, pero abunda en estrategias para ampliar el fanon; 2) contesta la pregunta común en los fanfics de universo alterno: ¿qué pasaría si ... ? y 3 ) es coherente con el subgénero Hurt/Comfort: Harry sufre mucho y el profesor Snape lo consuela.

Si se la analiza como ficción, es una historia bien contada. Tiene un tema principal: el significado de la diversidad, tratado a través de la visión personal: qué significa para Harry su diversidad. Luego, un significado en contexto: qué significa la diversidad para los individuos que se relacionan con él. Finalmente, institucional: qué significa para Hogwarts. El tema del bullying es ampliamente tratado en la historia.

Una estrategia que prevalece en la ficción es dotar algunas dosis de información que resultan educativas alrededor del TEA: cómo tratar a una persona con TEA en un momento de crisis, cuál es su origen, cuál es el peso de los aspectos sensoriales en la vida cotidiana de una persona con autismo, cómo es un espacio de terapia sensorial, etc. Pero lo más importante es un punto de vista que parece interno.

Por otro lado, mientras se podría haber esperado que Harry encarnara un savant, una persona con autismo con capacidades excepcionales, Harry es una persona con TEA con características promedio. A pesar de que en la historia se muestran algunas de sus capacidades excepcionales provenientes del canon, como ser un excelente volador con dominio sobre su escoba, su caracterización se modifica: a Harry solo le interesa volar y no le interesa atrapar the snitch, debido a que el juego le parece violento y peligroso. La estrategia general exitosa es el balance entre los aspectos narrativos de la historia, las alusiones claras al canon y una representación del TEA que hace pensar que el escritor está muy familiarizado con una persona dentro del espectro. Como se verá en la siguiente sección, las reacciones de los lectores parecen confirmarlo.

\section{Análisis de comentarios hechos a Different, Not Defective}

La mayoría de los comentarios tienen un tono emocional. Esto coincide con lo encontrado por Samutina (2016) en la comunidad rusa de fan fic- 
tion en torno a Harry Potter. Prácticamente, en su totalidad, los comentarios son muy positivos. Muchos expresan aprobación o agrado, sin dar más detalles. En algunos otros, se felicita al autor o se le pide que escriba más, y solo en algunas ocasiones se le comenta sobre aspectos narrativos. El tipo de vocabulario, la frecuencia de las felicitaciones y la intensidad emocional de las expresiones muestran que esta historia, más allá de un simple interés, generó pasión.

Del total de 863 comentarios de retroalimentación analizados, $25 \%$ se relaciona con el tema de autismo. Pero dentro de los quince capítulos este porcentaje varía. El capítulo 1 tiene $34 \%$ de comentarios relacionados, mientras que el capítulo 7 se eleva a $41 \%$.

Los retroalimentadores que más comentan este tema son en su mayoría personas relacionadas con el autismo: personas con TEA, familiares, voluntarios y especialistas. También hay comentarios de personas que no conocían el autismo.

El análisis cualitativo de los comentarios reveló los siguientes hallazgos:

Las evaluaciones de algunos de los lectores muestran que el texto es aprobado no solo a nivel emocional, sino en cuanto a la verosimilitud y el tratamiento del tema.

Los lectores comentan que temían encontrar estereotipos al ver que se trataría el autismo, pero que están gratamente sorprendidos de no encontrarlos. Esta sensación es compartida por un lector cuya madre es asistente educativa de niños con autismo y que considera que el texto coincide con la descripción de su madre. El lector añade: "Your portrayal of Harry is positively adorable, it makes me smile every time I read it. I really really like this fiction" [Tu retrato de Harry es positivo y adorable. Me hace sonreír cada vez que lo leo. Realmente me encanta esta historia].

Otro lector afirma haber conocido y trabajado con personas con autismo y señala: "This is an absolutely AWESOME story! [... ] you have done a 
wonderful job portraying both the challenge and the privilege it is to know them. Thank you so much for this. I look forward to more whenever you get the time" [Esta es una historia increíble [...] has hecho un increíble trabajo representando tanto el reto como el privilegio que es conocerlos. Muchas gracias por ello. Espero que escribas más cuando tengas tiempo].

Una madre afirma que el texto es como escuchar la historia de su propio hijo. Los lectores con TEA comparten esta visión expresando que se identifican con la caracterización del protagonista de la historia y evalúan muy positivamente la representación. Uno manifiesta: "I have Autism, more severe when I was younger, and this is one of the best interpretation I've read of Autism in a long while, not just in fan fiction" [Tengo autismo, más grave cuando era pequeño. Esta es una de las mejores interpretaciones que he leído sobre autismo no solo en fan fiction]. Otro comenta: "As an autistic person, there are very few books written with characters like me, and even fewer fanfictions. You do this justice... It's like starring into a mirror!” [Como una persona con autismo, siento que hay muy pocos libros escritos con personajes como yo, y menos aún fan fiction. Tú escribes del tema con justicia. Es como mirarme en un espejo].

La lectura tiene una función emocional para los lectores con TEA y para sus familias

Varios de los lectores confiesan que el texto los hizo llorar. Añaden información de su propia experiencia, con similitudes a la del protagonista: "Yeah, I do the stemming as well! [...] As for the intense interest portion: it has been and always will be Space! Whether it be the science of astronomy or science fiction, I can talk your ears off about it!" [Sí, yo también agito mis manos [... en cuanto a los intereses intensos para mí siempre ha sido y será el espacio, ya sea ciencia, astronomía o ciencia ficción. Puedo hablarte por horas de ello]. Agradecen haber encontrado el texto y reflejarse tanto como algo valioso para ellos como para que se represente el autismo desde una visión que perciben como verosímil y conmovedora a la vez: "This chapter made me cry. I was called a retard many times in my life. I love this. I laughed when I read about Harry monotone lecture of chemistry that's me with Harry Potter" [Este capítulo me hizo llorar. Muchas veces en mi vida me han llamado retrasado. Me encanta esta historia. Me reí cuando leí so- 
bre la monótona conferencia de Harry sobre química. Así soy yo con Harry Potter]. Otro agrega: "Oh God, this fic just keeps sending me back to memory lane [... ] As I look back, I thank God for how far I've come, and how much I've grown" [Dios mío, este fic continúa haciéndome regresar al pasado $[\ldots]$ conforme miro hacia atrás, agradezco a Dios por lo lejos que he llegado y lo mucho que he crecido]. Son significativos en este sentido los comentarios de familiares: "Absolutely beautiful story. My brother has autism and I've seen him struggle with the same things that Harry does. This story has already become near and dear to my heart" [Es una historia absolutamente bella. Mi hermano tiene autismo y lo he visto luchar con lo mismo con lo que Harry lucha. Esta historia me ha llegado al fondo del corazón].

El uso de las estrategias narrativas y la aproximación promovieron la concienciación y la sensibilización sobre el TEA. Diversos comentarios de personas que no conocían la condición muestran cómo aumentó su comprensión y su actitud: "I admit that I knew very little about autism before beginning this story. You are doing an excellent job of teaching us what it is without it feeling like a lecture on autism" [Admito que sabía muy poco sobre autismo antes de leer esta historia. Estás haciendo un excelente trabajo enseñándonos sobre el tema sin hacernos sentir como que recibimos una clase sobre autismo]. Otra aplaude que se ve que el autor sabe de autismo y apunta: "Es tan maravilloso el concepto de no ser roto, sino sencillamente diferente, me has cautivado, hermosa historia”.

\section{Conclusiones}

\section{Aportaciones en hallazgos: representación del autismo}

Fanfiction.net es un espacio de afinidad (Black, 2008) en el que los fans se apropian de sus ficciones favoritas, pero también expresan a través de la ficción temas de interés para ellos más allá de la ficción. Como ya lo decía Jenkins (1992), una de las estrategias narrativas es la personalización, a través de la cual el autor del fanfic aparece dentro de la ficción como un personaje más. Esta ha ampliado su sentido. En este caso, el protagonista de la ficción es Harry Potter, pero un Harry Potter que se transforma como personaje en la fusión con la personalidad y las características de 
la persona con autismo o con un familiar cercano que tiene la condición que posiblemente escribió la historia. Así, la personalización se vuelve una estrategia catártica y de autorrepresentación que lleva al fandom temas como la discapacidad, las condiciones médicas, el bullying y la aceptación por la diferencia.

En Different, Not Defective, el tema de lo que significa tener autismo se representa tanto en las ficciones como en la negociación del sentido de los retroalimentadores (Wenger, 2001). En esta historia, las personas con TEA o familiares añaden información sobre su propia experiencia de vida. No se puede decir que esto llega a una discusión, pues los comentarios no son siempre respondidos, pero este es un espacio donde las personas con TEA se expresan, descubren, se afirman como seres creativos, como lectores integrados en el fandom de sus cánones favoritos; sus expresiones los validan y descubren la diversidad en el TEA. Esta inclusión de las personas con TEA en estos espacios de afinidad y la inclusión del tema no parecen una estrategia planeada de representación ni derivarse de una intención activista. Sin embargo, influyen en la representación y en el diálogo entre personas con TEA, familiares y personas no relacionadas con el tema para conocer de primera mano qué significa vivir con TEA. Como señalan Jenkins (1992) y Fiske (1992) en el fandom, el proceso de recepción sucede siempre en comunidad. El fandom constituye una comunidad interpretativa y las prácticas de lectura de los fans deben entenderse más en términos institucionales que personales.

Es relevante descubrir cómo espacios de aparente función solo de entretenimiento para los fans tienen también una función social, lo cual coincide con lo encontrado por Black (2008). En su investigación, se destaca cómo el fan fiction sirve para aprender una segunda lengua y a la vez para afirmar la identidad y tener un autoconcepto positivo.

Los contrastes que existen en la representación del autismo en la ciencia, en los medios, en internet y en el fan fiction analizado coincide con lo observado por Sarret (2011) cuando afirma que parecen coexistir dos representaciones de la realidad autística: una que se basa en representaciones enraizadas 
en el discurso médico y enfocadas en el "déficit" que implica la "enfermedad" $\mathrm{y}$ otra que responde a narrativas y experiencias de vida de las personas con TEA y sus familias. El texto analizado pertenece a este segundo grupo.

Sarret (2011), Osteen (2010) y Quirici (2015) añaden que las mejores narrativas personales alrededor del autismo describen un largo proceso de descubrimiento y aceptación de una identidad no normativa, en lugar de un viaje hacia la cura o la recuperación. Esto puede verse en la caracterización de Harry y el viaje del héroe vivido por el protagonista de Different, Not Defective. Harry lucha por validarse y aceptarse a sí mismo.

Una de las cuestiones más interesantes reveladas en este texto es que no hay una separación entre autismo de bajo y alto funcionamiento; esto no se menciona literalmente y tampoco se connota en el texto. Se puede ver más una visión del TEA como un espectro y de cómo conviven las dificultades de Harry para atarse los zapatos a los 11 años, con su pasión y talento para la química. Es un ser menos estereotipado. De ahí se entiende la validación que hacen los lectores con TEA del texto y el papel emocional que desempeña su lectura según revelan sus reviews.

El sentido negociado sobre el autismo en comunidad apunta de manera distinta al activismo, por ser menos intencional, al menos en apariencia, en señalar las deficiencias de la representación médica o mediática. Pero al igual que el activismo tiene potencial de construir una representación, familiar, por parecida a nosotros, y valiosa, por singular. Así como lo señala Hall (2010), la representación alternativa se convierte en lucha ideológica de contra-poder. Esto permite afirmar la dignidad y los derechos de quienes - a través del discurso hegemonónico — han sido invisibilizados y estereotipados para mantener una idea unívoca de la normalidad.

Además de los hallazgos, se considera que este artículo tiene diferentes aportaciones.

En la metodología, utiliza la visualización de datos como estrategia para analizar narrativas y participación en plataformas. En los estudios de 
representación y de los estudios de discapacidad, amplía los corpus posibles a espacios de afinidad (Black, 2008) y de cultura participativa (Jenkins, 2006) donde el diálogo entre autor y lector es sincrónico; en los estudios de fan fiction al aportar categorías y estrategias de análisis para encontrar relaciones entre análisis narrativo y representación.

También se reconoce que, si bien este estudio analizó de manera profunda una ficción de 32000 palabras y 863 comentarios, está limitado a una sola representación. Las líneas futuras de investigación deberían cubrir el análisis narrativo de más ficciones de un solo canon, el contraste entre diversos cánones o el estudio de cómo se representan otras discapacidades.

\section{Referencias}

Acosta-Alzuru, C. (2013). Dear Micaela: Studying a telenovela protagonist with asperger's syndrome. Cultural Studies? Critical Methodologies, 13(2), 125-137.

American Psychiatric Association (2013). DSM-5. American Psychiatric Association.

Awesomelyglorious (2009-2011). Different, Not Defective. En FanFiction. Recuperado de https://www.fan fiction.net/s/7239464/1/Different-Not-Defective

Belcher, C. y Maich, K. (2014). Autism spectrum disorder in popular media: Storied reflections of societal views. Brock Education: A Journal of Educational Research and Practice, 23(2), 97-115.

Black, R. W. (2008). Adolescents and online fan fiction. Nueva York: Peter Lang.

Brownlow, C. (2010). Re-presenting autism: The construction of 'NT syndrome.' Journal of Medical Humanities, 31(3), 243-255.

Campbell, J. (1959). El héroe de las mil caras: psicoanálisis del mito. México: Fondo de Cultura Económica. 
Castillo, M.y Meneses, M. (2015). Dinámicas de comunicación y resistencia: el caso de \#Ayotzinapa en YouTube. En E. Nos, A. Arévalo y A. Farné, Comunicambio: comunicación y sociedad civil para el cambio social (pp. 757-769). Madrid: Fragua.

Dawson, M., Soulières, I., Gernsbacher, M. A. y Mottron, L. (2007). The level and nature of autistic intelligence. Psychological Science, 18(8), 657-662.

Fiske, J. (1992). The cultural economy of fandom. En L. A. Lewis (ed.), The adoring audience: Fan culture and popular media (pp. 30-49). Nueva York: Routledge.

Guerrero-Pico, M. del M. (2016). Historias más allá de lo filmado: Fan fiction y narrativas transmedia en series de televisión (Tesis de doctorado, Universidad Pompeu Fabra, Barcelona, España).

Hall, S. (2010). Sin garantías: trayectorias y problemáticas en estudios culturales. Popayán, Colombia: Envión Editores.

Heaton, P.y Wallace, G. L. (2004). Annotation: The savant syndrome. Journal of Child Psychology and Psychiatry, 45(5), 899-911.

Hellekson, K. y Busse, K. (eds.). (2006). Fan fiction and fan communities in the age of the Internet: New essays. Jefferson, NC: McFarland.

Hernández Sampieri, R., Fernández Collado C. y Baptista L. (2014). Metodología de la investigación. México: McGraw-Hill.

Jenkins, H. (1992). Textual poachers: Television fanes and participatory culture. Nueva York: Routledge.

Jenkins, H. (2006). Convergence culture: Where old and new media collide. Nueva York: New York University. 
Jones, S. C. y Harwood, V. (2009). Representations of autism in Australian print media. Disability \& Society, 24(1), 5-18.

Karpovich, A. I. (2006). The audience as editor: The role of beta readers in online fan fiction communities. En Hellekson, Ky Busse, K. (eds.), Fan fiction and fan communities in the age of the internet (pp. 171188). Jefferson, NC: McFarland.

Lugo, N. (2016). Diseño de narrativas transmedia para la transalfabetización (Tesis de doctorado, Universidad Pompeu Fabra, Barcelona, España).

Moretti, F. (2015). Lectura distante. México: Fondo de Cultura Económica.

Muñoz de la Llave, P. (19 abril 2011). Leo Kanner, el padre del autismo y de las "madres nevera". En Autismo Diario. Recuperado de https:// autismodiario.org/2011/04/19/leo-kanner-el-padre-del-autismoy-de-las-madres-nevera/

Osteen, M. (ed.) (2010). Autism and representation. Nueva YorK: Routledge.

Parrish, J. J. (2007). Inventing a universe: Reading and writing internet fan fiction (Tesis de doctorado, University of Pittsburgh, Pittsburgh, Estados Unidos).

Quirici, M. (2015). Geniuses without imagination: Discourses of autism, ability, and achievement. Journal of Literary \& Cultural Disability Studies, 9(1), 71-88.

Ramachandran, V. S. y Oberman, L. M. (2006). Broken mirrors: A theory of autism. Scientific American, 295(5), 62-69.

Samutina, N. (2016). Emotional landscapes of reading: Fan fiction in the context of contemporary reading practices. International Journal of Cultural Studies. 
Schwarz (2010). Film as vehicle for raising consciousness among autistic peers. En M. Osteen (ed.), Autism and representation. Nueva York: Routledge.

Sarrett, J. C. (2011). Trapped children: Popular images of children with autism in the 1960s and 2000s. Journal of Medical Humanities, 32(2), 141-153.

Stevenson, J. L., Harp, B. y Gernsbacher, M. A. (2011). Infantilizing autism. Disability Studies Quarterly, 31(3).

Thomas, B. (2011). What is fan fiction and why are people saying such nice things about it? Storyworlds: A Journal of Narrative Studies, 3(1), 1-24. doi: $10.1353 /$ stw.2011.0001

Vogler, C. (2007). The writer'sjourney. Los Ángeles: Michael Wiese Productions.

Wenger, E. (2001). Comunidades de práctica: aprendizaje, significado e identidad. Madrid: Paidós.

Wodka, E. L., Mathy, P. y Kalb, L. (2013). Predictors of phrase and fluent speech in children with autism and severe language delay. Pediatrics, 131(4), e1128-e1134. 